\title{
Embodied cognition and language learning in virtual environments
}

\author{
Yu-Ju Lan ${ }^{1} \cdot$ Nian-Shing Chen ${ }^{2} \cdot{\text { Ping } \mathrm{Li}^{3} \cdot \operatorname{Scott} \text { Grant }}^{4}$
}

Published online: 25 August 2015

(C) Association for Educational Communications and Technology 2015

\section{Introduction}

Based on the sociocultural theory of second language acquisition (SLA), it is the social context and interaction that mediate language learning; therefore, they have been considered significant in the SLA process (Ellis 2008). Additionally, the person and the world are connected in an inseparable relationship (Lantolf 2005). A context-dependent social interaction is most important to SLA because it provides L2 learners with essential scaffolding for acquiring an L2 (Vygotsky 1978). Swain (2000) suggested that language learning occurs both inside the head of the learner and in the world in which the learner

Yu-Ju Lan

yujulan@gmail.com;

http://tell.aclc.ntnu.edu.tw/index.php/en/

Nian-Shing Chen

nschen@mis.nsysu.edu.tw;

http://www.nschen.net

Ping Li

pul8@psu.edu;

http://blclab.org

Scott Grant

scott.grant@monash.edu;

http://profiles.arts.monash.edu.au/scott-grant/; http://virtualhanyu.com;

http://virtuallyenhancedlanguages.com

1 Department of Applied Chinese Language and Culture, National Taiwan Normal University, Taipei, Taiwan

2 Department of Information Management, National Sun Yat-sen University, Kaohsiung, Taiwan

3 Pennsylvania State University, State College, USA

4 Faculty of Arts, Monash University, Melbourne, Australia 
experiences the learning. In short, internal mediation (mental activity) is originated through external mediation (Ellis 2008).

What has been emphasized in the inseparability of external and internal mediation during context-dependent interaction in sociocultural SLA is in line with the argument of embodied cognition. The formative role of the environment (context) plays in the development of cognitive process (Cowart 2005) is emphasized, specifically on the "interaction between perception, action, the body and the environment" (Barsalou 2008), which is different from the traditional perspective that the body plays a small role in cognition. Studies in line with embodied cognition have observed different roles of actions in cognitive processes and have suggested that human mind is closely connected to sensorimotor experience. Several general theories of embodied cognition, such as those proposed by Glenberg et al. (Glenberg et al. 2004; Glenberg and Goldberg 2011; Glenberg and Kaschak 2002) and Barsalou (2008) argued that the cognitive process develops when a tightly coupled system emerges from interactions between organisms and their environment, with the interactions being real-time and goal-directed (Cowart 2005).

What has been found in terms of embodied language processing that a person's bodily sensations and actions will impact how he/she comprehends language is consistent with the indexical hypothesis which states that an understanding of language results from a simulation of the actions implied by the meaning of the sentence (Glenberg and Kaschak 2002). Accumulating evidence from embodied cognition research supports the argument that action enhances comprehension (Asher 1977; Glenberg and Goldberg 2011; Glenberg et al. 2004; Tellier 2008). In recent years, the findings obtained from brain research also echo the view that language processing is an embodied process (Aziz-Zadeh and Damasio 2008; Willems and Casasanto 2011); that bodily action in the contextual environment and the person's perceptual experiences are inseparable during the cognition process. Rueschemeyer et al. (Rueschemeyer et al. 2010) state that intentional actions activating the brain resources used for the motor system are also engaged in lexical-semantic processing and language comprehension. Additionally, the motor system is automatically activated under the following three situations: when a person (a) observes manipulable objects; (b) processes action verbs; and (c) observes the actions of another individual (Mahon and Caramazza 2008).

Virtual immersion environments, such as Second Life (SL, a multiuser virtual environment) or Massively Multiplayer Online Role-Playing Games (MMORPGs), have gained the attention of cross-disciplinary researchers (Lan 2014; Wang and Burton 2013) because they make both avatar-self movement and different interactions between the learner and the virtual environments possible (Lan et al. 2013). Thus, such environments have been recognized to provide learners with an embodied learning experience (Schubert et al. 1999). In contrast to controlling an avatar via a mouse or a keyboard (like SL and MMORPGs), gesture-based technologies (like Play Station Move and MS Kinect) that mainly involve gestures, or body motion, have also been widely used to support the physical effects on learning (Chao et al. 2013; Chang et al. 2013; Hung et al. 2014). However, the abovementioned embodied motion and the interaction obtained in a virtual environment (VE) are accomplished not via the learners' physical bodies, but their avatars. Thus, we may have to wonder whether the avatar-based embodied motions are sufficient and strong enough to originate the essential internal mediation in learners' brains and consequently have an effect on language comprehension and acquisition. Obviously, more cross-disciplinary evidence is needed to answer the abovementioned questions and to add to the knowledge base of embodied cognition and language learning in virtual worlds. 
This special issue aims at providing a platform for researchers to present their efforts on studies that may offer insights into the relationship between virtually embodied cognition and language acquisition. These are open questions worthy of further exploration. It is expected that through the publication of this special issue, we can help develop a further understanding of embodied cognition and language learning. After a rigorous review process, eight high-quality research papers have been accepted for publication in this special issue, and these papers clearly explain the relationship between embodied cognition and language learning in VEs from different perspectives. We hope that these studies will inspire future research in this direction.

In the first paper entitled "The effects of collaborative models in Second Life on French learning," Hsiao, Yang, and Chu investigated the effects of employing different collaborative models on learners' French performance and their perceptions about learning French. Twenty-three college students participated in the study. After analyzing studentcreated movies and students' collaborative process, it was found that by virtually involving the students in context-inclusive collaboration, their speaking capability and inter-peer collaboration can be positively influenced. In the second paper with the title of "Second language acquisition of Mandarin Chinese vocabulary: context of learning effects," Lan, Fang, Legault, and Li built three learning contexts (zoo, supermarket, and kitchen), in both the virtual and the traditional web-based environments, for learners of Chinese as a foreign language. Thirty-one monolingual English speakers, randomly assigned to the VE and the traditional learning contexts, participated in a training study in which they were learning 90 Chinese words. After the experiment, the participants' behavioral performance with regard to accuracy, reaction time, and exposure were collected and analyzed by using variance and mixed-effects modeling. The results showed a larger acceleration in the learning trajectory for the participants in the VE context than those in the traditional learning context. The results suggest that simulated embodied experience in the VE may have aided vocabulary acquisition in a second language. Huang and Huang confirmed the effect of embedding a scaffolding strategy in a handheld sensor-based vocabulary game on the learning motivation and performance of students of English as a foreign language. In their paper with the title of "A scaffolding strategy to develop handheld sensor-based vocabulary games for improving students' learning motivation and performance," they found that with the supports of scaffolding, the low-achieving students' motivation and performance in vocabulary learning improved significantly. Next, in the fourth paper, PasfieldNeofitou, Huang, and Grant investigated the relationship between virtual embodiment and language learning. Through two case studies, they found that the multimodal communication established by the participants in virtual worlds made the distinguishability between participants' real identities and their virtual avatars vague. In other words, the boundaries between the real and VE are highly permeable, with students moving freely between the two, and the actions of their avatars in the VE having a direct and real cognitive impact on the students themselves.

In the fifth paper, Lin, Chao, and Huang investigated the effects of one of the most discussed affective factors, anxiety, on college students' learning of the Japanese language. They developed an intelligent affective tutoring system to recognize the facial expressions of learners of Japanese language for providing them with adequate feedback. The results show that this proposed system is beneficial for the learning of the Japanese language, reducing learning anxiety, and improving learning effectiveness. Different from previous papers with general languages as the target languages (Chinese, English, French, or Japanese), in the sixth paper, Hung, Hsu, Chen, and Kinshuk investigated the effects of a situated embodiment-based strategy with flag semaphore on learning sign languages. A 
total of 60 college students with no experience in learning flag semaphore participated in their study. The results show that the proposed strategy with situated embodiment-based learning effectively improved participants' procedural knowledge construction and enhanced their attention level with the lower extrinsic cognitive load in the learning process. In the seventh paper entitled "Vocabulary learning in massively multiplayer online games: context and action before words," Zheng, Bischoff, and Gilliland investigated how vocabulary was learnt by FL learners in Word of Warcraft (WOW), a massively multiplayer online game by analyzing both chat and avatar action data obtained from a 2-h co-play between two players. The analytic results provide the readers with an alternative explanation of how players embodied in their avatars appropriated semiotic resources imbued in WOW and make vocabulary learning salient in this context. Last but not least, in the eighth paper, Hong, Hwang, Tai, and Lin, investigated the relationship among selfefficacy, competitive anxiety, and gameplay interest of elementary students in a one-onone competition setting. A total of 278 fifth and sixth graders participated in their study. The results show that both self-efficacy and gameplay interest are negatively associated with students' competitive anxiety.

The abovementioned papers will likely provide readers with a deep and extensive understanding of the relationship between embodied cognition and language learning in VEs. The covered target languages include Chinese, English, French, Japanese, sign, and non-verbal. Moreover, the participants were drawn from a range of ages, from elementary school students to college students. The investigated variables relating to language learning are various, including performance, motivation, anxiety, self-efficacy, and sense of identity. Although the papers included in this special issue have covered a broad range of issues in embodied cognition and language learning in virtual worlds, there are other issues that may further attract researchers' attention in the future. For example, longer-term longitudinal studies, rather than studies in short terms like several hours, days, or 1 or 2 months, are needed. Additionally, the analysis of learners' learning behaviors in virtual worlds relating to SLA is essential, such as their communication patterns and strategy usage. As argued by Lan (2015), learning a language in virtual worlds can improve learners' performance, motivation, as well as provide them with authentic contexts for conducting embodied and game-liked learning activities that meet people's growing experiences in the digital era. Researchers' efforts on exploring the potential of virtual worlds for SLA will be worthwhile because it presents a potential solution to the problems encountered in today's second language education.

\section{References}

Asher, J. T. (1977). Learning another language through actions: The complete teacher's guidebook. Los Gatos: Sky Oaks Productions.

Aziz-Zadeh, L., \& Damasio, A. (2008). Embodied semantics for actions: Findings from functional brain imaging. Journal of Physiology, 102, 35-39.

Barsalou, L. W. (2008). Grounded cognition. The Annual Review of Psychology, 59, 617-645. Retrieved from http://www.cogsci.ucsd.edu/ ajyu/Teaching/Cogs202_sp12/Readings/barsalou08_grounded. pdf.

Chang, C. Y., Chien, Y. T., Chiang, C. Y., Lin, M. C., \& Lai, H. C. (2013). Embodying gesture-based multimedia to improve learning. British Journal of Educational Technology, 44(1), E5-E9.

Chao, K. J., Huang, H. W., Fang, W. C., \& Chen, N. S. (2013). Embodied play to learn: Exploring Kinectfacilitated memory performance. British Journal of Educational Technology, 44(5), E151-E155. 
Cowart, M. (2005). Embodied cognition. Retrieved April 23, 2014, from http://www.iep.utm.edu/ embodcog/.

Ellis, R. (2008). The study of second language acquisition (2nd ed.). New York: Oxford University Press.

Glenberg, A. M., \& Goldberg, A. B. (2011). Improving early reading comprehension using embodied CAI. Instruction Science, 39, 27-39.

Glenberg, A. M., Gutierrez, T., Levin, J. R., Japuntich, S., \& Kaschak, M. P. (2004). Activity and imagined activity can enhance young children's reading comprehension. Journal of Educational Psychology, 96(3), 424-436.

Glenberg, A. M., \& Kaschak, M. P. (2002). Grounding language in action. Psychnomic Bulletin \& Review, 9(3), 558-565.

Hung, I.-C., Lin, L.-I., Fang, W.-C., \& Chen, N.-S. (2014). Learning with the body: An embodiment-based learning strategy enhances performance of comprehending fundamental optics. Interacting with Computers,. doi:10.1093/iwc/iwu011.

Lan, Y. J. (2014). Does second life improve Mandarin learning by overseas Chinese students? Language Learning and Technology, 18(2), 36-56.

Lan, Y. J. (2015). Contextual EFL learning in a 3D virtual environment. Language Learning and Technology, 19(2), 16-31.

Lan, Y.-J., Kan, Y.-H., Hsiao, I. Y. T., Yang, S. J. H., \& Chang, K.-E. (2013). Designing interaction tasks in second life for Chinese as a foreign language learners: A preliminary exploration. Australasian Journal of Educational Technology, 29(2), 184-202.

Lantolf, J. (2005). Sociocultural and second language learning research: an exegesis. In E. Hinkel (Ed.), Handbook of research on second language teaching and learning. Mahway: Lawrence Erlbaum.

Mahon, B. Z., \& Caramazza, A. (2008). A critical look at the embodied cognition hypothesis and a new proposal for grounding conceptual content. Journal of Physiology Paris, 102, 59-70.

Rueschemeyer, S. A., Lindemann, O., van Rooij, D., van Dam, W., \& Bekkering, H. (2010). Effects of intentional motor actions on embodied language processing. Experimental Psychology, 57(4), 260-266.

Schubert, T., Friedmann, F., \& Regenbrecht, H. (1999). Embodied presence in virtual environments. In R. Paton \& I. E. Neilson (Eds.), Visual representations and interpretations. Berlin: Springer.

Swain, M. (2000). The output hypothesis and beyond: mediating acquisition through collaborative dialogue. In J. Lantolf (Ed.), Sociocultural theory and second language learning. Oxford: Oxford University Press.

Tellier, M. (2008). The effect of gestures on second language memorisation by young children. Gesture, $8(2), 219-235$.

Vygotsky, L. (1978). Mind in society: The development of higher psychological process. Cambridge: Harvard University Press.

Wang, F., \& Burton, J. K. (2013). Second Life in education: A review of publications from its launch to 2011. British Journal of Educational Technology, 44(3), 357-371.

Willems, R. M., \& Casasanto, D. (2011). Flexibility in embodied language understanding. Frontiers in Psychology, 2, 116.

Yu-Ju Lan is currently a Distinguished Professor in the Department of Applied Chinese Language and Culture at National Taiwan Normal University. Before becoming a professor at the university, she had been teaching in two different elementary schools in Taiwan for more than 20 years. She enjoys working with children and loves to help them grow and learn. This passion has motivated her to build a wide variety of learning environments to meet Chinese as foreign language and English as foreign language learners' multiple needs. She served as Chair of the Special Interest Group: Technology Enhanced Language Learning in the Asia-Pacific Society for Computers in Education from 2012 to 2013. Her research interests include language learning in virtual worlds, mobile learning, and online synchronous teacher training. She has been working with a group of researchers to develop and implement the curriculum, pedagogy, lessons, teaching materials, etc., based on the online 3D virtual contexts and mobile learning environments for foreign students learning a language or culture. For more information about Lan's research please consult http://tell.aclc.ntnu.edu.tw/index.php/en/.

Nian-Shing Chen is Chair Professor in the Department of Information Management at National Sun Yat-sen University, Taiwan. He has published over 400 papers in the international referred journals, conferences and book chapters. One of his papers published in Innovations in Education and Teaching International was awarded as the top cited article in 2011. He is an author of three books with one textbook entitled "eLearning Theory \& Practice". Prof. Chen received two outstanding research awards from the National 
Science Council, Taiwan in 2008 and 2011-2013. His current research interests include assessing e-Learning course performance; online synchronous teaching \& learning; mobile \& ubiquitous learning; Game-based learning and Cognition \& Gesture-based learning. Prof. Chen is serving as an editorial board member for many international journals and guest editors for special issues of international journals. He has also organized and chaired numerous international conferences and workshops in the area of advanced learning technologies. Professor Chen is a senior member of IEEE, ACM and the current Chair for the IEEE Technical Committee on Learning Technology (http://lttf.ieee.org/). He is Editor-In-Chief of the SSCI indexed Journal of Educational Technology \& Society.

Ping Li is Professor of Psychology, Linguistics, and Information Sciences and Technology at the Pennsylvania State University, where he is also Associate Director of the Institute for CyberScience, CoDirector of the Center for Brain, Behavior, and Cognition, and Co-Director of the Advanced Joint Center for the Study of Learning Sciences. His research interests are in the cognitive science and cognitive neuroscience of language acquisition and bilingualism, and his publications include co-authored books such as Acquisition of Lexical and Grammatical Aspect; Handbook of East Asian Psycholinguistics; the Psycholinguistics of Bilingualism; and Having Success with NSF: A Practical Guide. He is currently Editorin-Chief of the Journal of Neurolinguistics and Associate Editor of Frontiers in Psychology: Language Sciences. He has previously served as Editor of Bilingualism: Language and Cognition, President of the Society for Computers in Psychology, and Director for the Cognitive Neuroscience Program and the Program in Perception, Action, and Cognition at the National Science Foundation. For more information about Li's research please consult http://blclab.org/.

Scott Grant is the Coordinator of Chinese Introductory and Chinese Online Media in the Faculty of Arts at Monash University, Australia. He has been teaching Chinese as a foreign language at tertiary level for 20 years and is creator of the Monash Chinese Island 3D multiuser virtual environment (3D MUVE) in Second Life that has been part of the beginner level Chinese language curriculum at Monash since 2008. In 2012 he obtained two seed grants from the Languages and Culture Network for Australian Universities for the development of an immersive Arabic language and culture 3D MUVE and for research into foreign language anxiety. In 2013/14 was the initiator and Project Team Leader of an Australian Government funded project aimed at creating freely shareable pedagogical and technical resources for task-based language learning in the 3D MUVEs of Second Life and OpenSim. He has published on educational uses of 3D MUVEs on topics including self-efficacy, cognitive skills, second language acquisition and foreign language anxiety. 